\title{
Myxomycete research in the Philippines: Updates and opportunities
}

\author{
Dagamac NHA ${ }^{1}$ and dela Cruz TEE ${ }^{2}$ \\ ${ }^{1}$ Institute of Botany and Landscape Ecology, University of Greifswald, Soldmannstr. 15, D-17487, Greifswald, \\ Germany \\ ${ }^{2}$ Department of Biological Sciences, College of Science and Fungal Biodiversity and Systematics Group, Research \\ Center for the Natural and Applied Sciences, University of Santo Tomas, España 1008 Manila, Philippines
}

Dagamac NHA, dela Cruz TEE. 2015 - Myxomycetes research in the Philippines: Updates and opportunities. Mycosphere 6(6), 784-795, Doi 10.5943/mycosphere/6/6/12

\begin{abstract}
Myxomycetes, commonly known as slime molds, are phagotrophic, eukaryotic organisms that exhibit both fungal and protozoan characteristics. They are widely distributed both in temperate and tropical ecoregions, where they usually occur on dead plant substrates, such as bark, twigs, dried leaves, woody vines, and even decayed inflorescences or fruits. Their unique, diverse morphologies and fascinating life strategies make them ideal model organisms to study life processes. However, despite the high potential diversity in tropical systems, little is known about them particularly in archipelagic countries, such as the Philippines. In fact, previous studies on myxomycetes in the Philippines in the late 70s and early 80s by Reynolds encompassed the most comprehensive listing for the country. A total of 107 species were recorded at that time for the Philippines and roughly 50\% of these species represented new records for the country. But the paper was mainly an extensive, annotated species listing. In recent years, myxomycete research in the country has progressed beyond species lists to diversity and ecological studies. Several papers by the UST RCNAS Fungal Biodiversity and Systematics group have documented the occurrence and distribution of slime molds in several habitat types, e.g. in forest parks, coastal and inland limestone forests, lowland mountain forests, and from varied substrata - grass litter, aerial and ground leaf litter, twigs, and bark. These studies updated the list of species of myxomycetes in the Philippines to 150. These also provided baseline information on the ecological patterns and geographic distribution of slime molds in the tropics. This paper presents an update on slime mold research in the Philippines for the 35 years following Reynolds' publication in 1981 and discusses challenges and opportunities for further studies.
\end{abstract}

Key words - archipelago - checklist - Paleotropics - slime molds - taxonomy

This paper is written in honor of two mycologists who started and contributed significantly to the myxomycete research in the Philippines, Don Reynolds and Irineo J. Dogma Jr.

\section{Introduction}

Myxomycetes, also known as plasmodial slime molds or true slime molds, are a small, relatively homogenous group of phagotrophic fungus-like protists (Schnittler et al. 2012, Rojas \& Stephenson 2013), with about 1000 species known and described worldwide (Lado 2005-2015). These species are often abundant in many forested regions, particularly in areas with decaying logs, 
twigs and leaf litter (Stephenson \& Stempen 1994, Rojas \& Stephenson 2013). These substrata provide them with a sufficient supply of potential food, such as bacteria, yeasts, algae and other microscopic organisms (Lado et al. 2013). Myxomycetes are also recognized as producers of bioactive secondary metabolites. For example, Dembitsky et al. (2005) listed about 100 natural compounds, e.g. fatty acids, lipids, napthoquinone pigments, aromatic compounds, alkaloids and terpenoids, isolated and obtained from several species of myxomycetes. Several of these compounds are known to exhibit antitumor activities, e.g. cyclic phosphatidic acid from Physarum polycephalum (Kobayashi et al. 2002) and pyrroloiminoquinone from Didymium bahiense (Ishibashi et al. 2001). Myxomycetes are also key components in soil microbiota (Feest \& Madelin 1988, Stephenson et al. 2011, Hoppe \& Schnittler 2015) and may play a role in maintaining balance in soil ecosystems (Feest 1987; Foissner 1999). Many insects also depend on slime molds for food (Keller \& Snell 2002). Despite their numerous applications and important role in nature, the study of the biodiversity of myxomycetes has not been given much attention particularly in the Southeast Asian Paleotropics such as the Philippines, where higher species diversity can be expected (Dagamac et al. 2014).

The earliest report of myxomycetes in the Philippines started from Uyenco (1973) who originally claimed to have published the first report on Philippine myxomycetes. From the 314 specimens collected by Uyenco from Luzon (Quezon City and Laguna) and Mindanao (Basilan and Zamboanga) during the year 1961 to 1973,18 species were reported belonging to 10 genera. However, Dogma (1975) noted that Martin \& Alexopoulos (1969) already credited the Philippines with 22 species in their book entitled "The Myxomycetes". Dogma (1975) then listed 46 species of myxomycetes from 20 genera for the Philippines. The most wide-ranging annotation on Philippine myxomycetes however, was conducted by Don Reynolds in 1981. Reynolds (1981) initially reported the myxomycete collections from Mindanao (Davao, Cotabato and Zamboanga) by E. B. Copeland, and from Luzon by A. D. E. Elmer (Benguet) and E.D. Merrill (Bataan, Manila, Cavite and Laguna). These collections can now be found in the British Museum in London. He also presented an annotated list of 107 species of Philippine myxomycetes based on published and unpublished records. In this list, 53 species were listed as novel records for the country. Myxomycetes that are part of the species list were from the genera Arcyria, Badhamia, Ceratiomyxa, Clastoderma, Comatricha, Craterium, Cribaria, Diachea, Dictydium, Diderma, Didymium, Echinostelium, Fuligo, Hemitrichia, Lamproderma, Licea, Lycogala, Metatrichia, Perichaena, Physarella, Physarum, Stemonitis, Trichia, and Tubifera. Most of these myxomycetes were collected from substrates obtained from several sites in the country: (1) Luzon: Batanes, Ilocos, Mt. Province, Benguet, Bataan, Laguna, Quezon and Palawan, (2) Visayas: Camarines Sur, Sorsogon, Albay, Iloilo, Antique, Leyte, Negros and Cebu, and (3) Mindanao: Agusan, Cotabato, Surigao del Sur, Davao, Zamboanga and Sulu. The 107 myxomycete species reported in this study also included one unidentified species of Didymium collected from Cebu that resembles $D$. squamulosum, except that the spores are larger than the typical D. squamulosum. Moreover, two myxomycete species listed were currently synonyms of two other myxomycete species found on that same list. Stemonitis nigrescens and $S$. smithii are basionyms of S. axifera and $S$. fusca, respectively. The list also corresponded to $25 \%$ of known species at that time and to $60 \%$ of the estimated total myxomycete flora in the Philippines (Reynolds, 1981). Since then, myxomycete survey remained stagnant.

For several decades, there were no published literatures about any myxomycete records since the last comprehensive annotation of Reynolds (1981). Thus in 2009, dela Cruz et al. initially looked at the myxomycetes collection at the Museum of Natural History Mycological Herbarium at the University of the Philippines in Los Baños, Laguna. Myxomycete specimens here were mainly deposited by Dogma, Reynolds and Quimio and belonged to the 21 genera: Arcyria, Ceratiomyxa, Cienkowskia (now identified as Willkommlangea), Clastoderma, Comatricha, Craterium, Cribaria, Diachea, Dictydium, Diderma, Didymium, Fuligo, Hemitrichia, Lamproderma, Lycogala, Perichaena, Physarella, Physarum, Stemonitis, Trichia and Tubifera. Interestingly, the collection also included specimens from the USA, Ecuador and Costa Rica dating from pre-World War II, i.e. 
1888 to 1939 . These deposited specimens were found to be generally in good condition. On the same year, a specimen found in Anda Island, Pangasinan was identified as a new species, Craterium retisporum (Moreno et al. 2009). This new myxomycete species were morphologically erected as new to science because of its distinguishing prominent reticulations found on the spore ornamentation. Dagamac et al. (2010) added five new records of myxomycetes for the Philippines, i.e. Clastoderma microcarpum, Dianema harveyi, Diderma subasteroides, Physarum leucophaeum, and Stemonitis pallida. These species were collected from bark samples of Samanea samans (Jacq) Merr. obtained from 21 different sites in Luzon Island. Furthermore, Dagamac et al. (2011) reported another 5 new records, namely, Arcyria afroalpina, Collaria arcyrionema, Craterium concinnum, Enerthenema papillatum, and Licea biforis from Mount Arayat National Park, Pampanga. Two additional records, Lepidoderma tigrinum and Perichaena pedata, were also reported by dela Cruz et al. (2011) as new records for the Philippines. Macabago et al. (2012) reported seven newly recorded species of myxomycetes in the Philippines which were identified as Arcyria globosa, Collaria rubens, Comatricha robusta, Craterium atrolucens, Lamproderma cacographicum, Oligonema schweinitzii, and Perichaena microspora from Lubang Island, Occidental Mindoro. Additionally, three new records were reported by Kuhn et al. (2013a) from Pangasinan and additional 17 new records by dela Cruz et al. (2014) from Bataan, Cavite and Subic. Recently, Dagamac et al. (2015a) found 8 new records in Bicol Peninsula which now brings the total of myxomycetes recorded in the Philippines to 150.

With such additions to the myxomycete flora accounted for a biodiversity hotspot like the Philippines, this paper (1) provides an updated checklist of the slime molds reported in the Philippines and (2) discusses the challenges and opportunities of engaging one's interest in myxomycete research in the country.

\section{General Study Area}

The Philippines is an archipelago composed of 7,107 islands that lies in the Southeast Asian region. Majority of these islands are assumed to be volcanic in origin as it is geographically part of the Pacific Ring of Fire (Hall 2002). General topography in the area is hilly and mountainous typically having constricted coastal plains with abundant rivers, streams and lakes (Catibog-Sinha \& Heaney 2006). Every island is endowed with sand beaches, clear coast lines and tropical rain forested mountains wherein the highest point in the country is the peak of Mt. Apo in Mindanao which is ca. 2,954 masl. The country's climate can be described to have a relatively high temperature and humidity with plentiful amount of annual rainfall (Lantican 2001). Similar to other tropical countries, the seasonality in the Philippines is divided into wet and dry seasons. Due to erratic rainfall distribution in different areas in the Philippines, four types of climate were designated on different parts of the archipelago (Philippine Institute for Development Studies, 2005). Type I are characterized of having two pronounced season that is dry during November to April, and wet during all the other months of the year. Areas included in this climate type are part of the Northwestern Luzon (Ilocos region, Western Mt. Provinces, and some part of the Southern Tagalog region). Type II climate has no clear dry period but with a very pronounced rainy season from the months of December to February. There is no single dry month and the minimum annual rainfall occurs during the months of March to May. The Southeastern Luzon areas (Bicol region and Quezon Province) are some examples of localities exposed to such climate type. Type III has no clear seasonality, but is relatively dry from November to April. Areas in the Philippines that experiences such climate type includes the northeastern part of the Ilocos region, scattered islands of the Central Visayas (Aklan, Capiz, Iloilo, Siquijor) and Western part of Mindanao (Agusan, del Sur, Bukidnon, and east Maguindanao). Lastly, Type IV climate is defined to have more or less an evenly distributed rainfall throughout the year and major Visayas islands like Cebu, Bohol, Western Samar and Southern Mindanao (Zamboanga, Davao, Sultan Kudarat) typically experience this climate.

Due to such promising topography and tropical climate distribution in the Philippines, the country is popularly known to be a biodiversity megahotspot (Catibog-Sinha \& Heany 2006). With 
its tropical climate, the country is gifted with numerous forest ecosystems: lowland rainforest, montane - mossy forest, pine forest and coastal, beach or mangrove forest. It is also completely bordered by tropical seas, thus isolating the archipelago from other Asian landmass by hundreds of kilometers of open water. Geological evidence has shown that the Philippines, with the exception of the Palawan and Mindoro regions, have always been isolated (Heaney 1998). Such geographic isolation and ideal climatic conditions resulted in floral and faunal endemicity, as high as $80 \%$ (Catibog-Sinha \& Heaney 2006). However, with its annual deforestation rate estimated to be $1.4 \%$ or about 89,000 hectares removed per year, the country is now listed as one of the most threatened ecosystems on the planet. In 2004, only $24 \%$ of the total land area (only about 7.2 million hectares of about 30 million hectares) remained covered with forests (Catibog-Sinha \& Heaney 2006). Thus, this necessitates an urgent assessment of the country's biodiversity since many areas remained unor under-explored and many species remain undiscovered including the myxomycetes.

\section{List of myxomycetes for the Philippines}

Table 1 In the myxomycete listing that follows, we present a table for all myxomycetes recorded for the Philippines. Information is provided on the source(s) of each record where the species was first mentioned, along with some general comments. Nomenclature basically follows Lado (20052015).

\begin{tabular}{|c|c|c|}
\hline & Myxomycete species & Sources \\
\hline 1 & Alwisia bombarda Berk. \& Broome & $\begin{array}{l}\text { First reported as Tubifera bombarda (Berk. \& } \\
\text { Broome) G.W. Martin by Reynolds (1981) }\end{array}$ \\
\hline 2 & Arcyria afroalpina Rammeloo & First reported from Dagamac et al. (2011) \\
\hline 3 & Arcyria cinerea (Bull.) Pers. & First reported from Reynolds (1981) \\
\hline 4 & Arcyria denudata (L.) Wettst. & First reported from Reynolds (1981) \\
\hline 5 & Arcyria globosa Schwein. & First reported from Macabago et al. (2012) \\
\hline 6 & Arcyria incarnata (Pers. ex J.F. Gmel.) Pers. & First reported from Reynolds (1981) \\
\hline 7 & Arcyria insignis Kalchbr. \& Cooke & First reported from Reynolds (1981) \\
\hline 8 & Arcyria magna $\mathrm{Rex}$ & First reported from Reynolds (1981) \\
\hline 9 & $\begin{array}{l}\text { Arcyria marginoundalata Nann.-Bremek. \& Y. } \\
\text { Yamam. }\end{array}$ & First reported from dela Cruz et al. (2014) \\
\hline 10 & Arcyria obvelata (Oeder) Onsberg & $\begin{array}{l}\text { First reported as Arcyria nutans (Bull.) Grev. by } \\
\text { Reynolds (1981) }\end{array}$ \\
\hline 11 & Arcyria virescens $\mathrm{G}$. Lister & First reported from Reynolds (1981) \\
\hline 12 & Badhamia affinis Rostaf. & First reported from dela Cruz et al. (2014) \\
\hline 13 & Badhamia utricularis (Bull.) Berk. & First reported from Reynolds (1981) \\
\hline 14 & Calomyxa metallica (Berk.) Nieuwl. & First reported from Reynolds (1981) \\
\hline 15 & Ceratiomyxa fruticulosa (O.F. Müll.) T. Macbr. & First reported from Reynolds (1981) \\
\hline 16 & Clastoderma debaryanum A. Blytt & First reported from Reynolds (1981) \\
\hline 17 & Clastoderma microcarpum (Meyl.) Kowalski & First reported from Dagamac et al. (2010) \\
\hline 18 & Collaria arcyrionema (Rostaf.) Nann.-Bremek. ex Lado & $\begin{array}{l}\text { First reported from Dagamac et. al. (2011) however } \\
\text { Reynolds (1981) first reported this species as } \\
\text { Lamproderma arcyrionema }\end{array}$ \\
\hline 19 & Collaria rubens (Lister) Nann.-Bremek. & First reported from Macabago et al. (2012) \\
\hline 20 & Comatricha fragilis Meyl. & First reported from Dagamac et al. (2015a) \\
\hline 21 & Comatricha longipila Nann.-Bremek. & First reported from Reynolds (1981) \\
\hline 22 & Comatricha nigra (Pers. ex J.F. Gmel.) J. Schröt. & First reported from Reynolds (1981) \\
\hline 23 & Comatricha pulchella (C. Bab.) Rostaf. & First reported from Dagamac et al. (2015a) \\
\hline 24 & $\begin{array}{l}\text { Comatricha robusta (T.N. Lakh. \& K.G. Mukerji) } \\
\text { Nann.-Bremek. \& Y. Yamam. }\end{array}$ & First reported from Macabago et al. (2012) \\
\hline 25 & Comatricha tenerrima (M.A. Curtis) G. Lister & Firs \\
\hline 26 & Craterium atrolucens Flatau & First reported from Macabago et al. (2012) \\
\hline 27 & Craterium concinnum $\operatorname{Rex}$ & First reported from Dagamac et. al. (2011) \\
\hline 28 & Craterium leucocephalum (Pers. ex J.F. Gmel.) Ditmar & Reynolds (1981) \\
\hline 29 & $\begin{array}{l}\text { Craterium microcarpum H.Z. Li, Yu Li \& Shuang L. } \\
\text { Chen }\end{array}$ & First reported from Kuhn et al. (2013a) \\
\hline 30 & Craterium minutum (Leers) Fr. & First reported from Reynolds (1981) \\
\hline 31 & Craterium paraguayense (Speg.) G. Lister & First reported from Reynolds (1981) \\
\hline
\end{tabular}




\section{Myxomycete species}

Craterium retisporum G. Moreno, D.W. Mitch. \& S.L. Stephenson

\section{Cribraria atrofusca G.W. Martin \& Lovejoy}

Cribraria cancellata (Batsch) Nann.-Bremek.

Cribraria microcarpa (Schrad.) Pers.

Cribraria piriformis Schrad.

Cribraria violacea Rex

Diachea bulbillosa (Berk. \& Broome) Lister

Diachea leucopodia (Bull.) Rostaf.

Diachea radiata $\mathrm{G}$. Lister \& Petch

Diachea splendens Peck

Dianema harveyi Rex

Dictydiaethalium plumbeum (Schumach.) Rostaf.

Diderma chondrioderma (de Bary \& Rostaf.) G. Lister

Diderma effusum (Schwein.) Morgan

Diderma fallax (Rostaf.) E.Sheld.

Diderma hemisphaericum (Bull.) Hornem.

Diderma rugosum (Rex) T. Macbr.

Diderma saundersii (Berk. \& Broome ex Massee) E. Sheld.

Diderma subasteroides M.L. Farr

Didymium anellus Morgan

Didymium anellus Morgan

Didymium clavus (Alb. \& Schwein.) Rabenh.

Didymium flocossum G.W. Martin, K.S. Thind \& Rehill

Didymium iridis (Ditmar) Fr.

Didymium leoninum Berk. \& Broome

Didymium megalosporum Berk. \& M.A. Curtis

Didymium melanospermum (Pers.) T. Macbr.

Didymium minus (Lister) Morgan

Didymium nigripes (Link) Fr.

Didymium ochroideum G. Lister

Didymium serpula Fr.

Didymium squamulosum (Alb. \& Schwein.) Fr. \& Palmquist

Didymium verrucosporum A.L. Welden

Echinostelium minutum de Bary

Elaeomyxa miyazakiensis (Emoto) Hagelst.

Enerthenema papillatum (Pers.) Rostaf.

Fuligo aurea (Penz.) Y.Yamam.

Fuligo cinerea (Schwein.) Morgan

Fuligo septica (L.) F.H. Wigg.

Hemitrichia calyculata (Speg.) M.L.Farr

Hemitrichia intorta (Lister) Lister

Hemitrichia leiocarpa (Cooke) Lister

\section{Hemitrichia minor G. Lister}

\section{Hemitrichia serpula (Scop.) Rostaf. ex Lister}

Lamproderma cacographicum Bozonnet, Mar. Mey. \& Poulain

Lamproderma scintillans (Berk. \& Broome) Morgan

Lepidoderma tigrinum (Schrad.) Rostaf.

Licea biforis Morgan
Sources

First reported from Moreno et al. (2009). This new species was described from collections in the Philippines and Western Australia.

First reported from Reynolds (1981)

First reported as Dictydium cancellatum (Batsch) T. Macbr. by Reynolds (1981)

First reported from Reynolds (1981) as Cribraria pachydictyon Nann.-Bremek.

First reported from Reynolds (1981)

First reported from Reynolds (1981)

First reported from Reynolds (1981)

First reported from Reynolds (1981)

First reported from Reynolds (1981)

First reported from Reynolds (1981)

First reported from Dagamac et al. (2010)

First reported from Reynolds (1981)

First reported from dela Cruz et al. (2014)

First reported from Reynolds (1981)

First reported as Diderma lyallii (Massee) T. Macbr. by Reynolds (1981)

First reported from Reynolds (1981)

First reported from Reynolds (1981)

First reported from dela Cruz et al. (2014)

First reported from Dagamac et al. (2010)

First reported from Reynolds (1981)

First reported from dela Cruz et al. (2014)

First reported from Reynolds (1981)

First reported from Dagamac et al. (2015a)

First reported from Reynolds (1981)

First reported from Reynolds (1981)

First reported from Reynolds (1981)

First reported from Reynolds (1981)

First reported from Reynolds (1981)

First reported from Reynolds (1981)

First reported from dela Cruz et al. (2014)

First reported from dela Cruz et al. (2014)

First reported from Reynolds (1981)

First reported from Dagamac et al. (2015a)

First reported from Reynolds (1981)

First reported from Kuhn et al. (2013a)

First reported from Dagamac et al. (2011)

First reported as Erionema aureum Penz. by Reynolds (1981)

First reported from dela Cruz et al. (2014)

First reported from Reynolds (1981)

First reported from Dagamac et al. (2015a) however Reynolds (1981) first reported this species as Hemitrichia stipitata (Massee) T. Macbr

First reported from Reynolds (1981)

First reported as Arcyria leiocarpa (Cooke) Massee by Reynolds (1981)

First reported from Dagamac et al. (2015a) however Reynolds (1981) first reported this species as Perichaena minor (G. Lister) Hagelst.

First reported from Reynolds (1981)

First reported from Macabago et al. (2012)

First reported from Reynolds (1981)

First reported from dela Cruz et al (2011)

First reported from Dagamac et al. (2011) 


\begin{tabular}{|c|c|c|}
\hline & Myxomycete species & Sources \\
\hline 80 & Licea erecta K.S. Thind \& Dhillon & First reported from Reynolds (1981) \\
\hline & & First reported as Licea floriformis var. aureospora \\
\hline 81 & Licea floriformis T.N. Lakh. \& R.K. Chopra & $\begin{array}{l}\text { M.T.M. Willemse \& Nann.-Bremek by dela Cruz et } \\
\text { al. (2014) }\end{array}$ \\
\hline 82 & Licea operculata (Wingate) G.W. Martin & First reported from dela Cruz et al. (2014) \\
\hline 83 & Lycogala epidendrum (L.) Fr. & First reported from Reynolds (1981) \\
\hline 84 & Lycogala exiguum Morgan & First reported from Reynolds (1981) \\
\hline 85 & Meriderma cribrarioides (Fr.) Mar.Mey. \& Poulain & $\begin{array}{l}\text { First reported as Lamproderma cribrarioides (Fr.) } \\
\text { R.E. Fr. By Reynolds (1981) }\end{array}$ \\
\hline 86 & $\begin{array}{l}\text { Metatrichia vesparia (Batsch) Nann.-Bremek. ex G.W. } \\
\text { Martin \& Alexop. }\end{array}$ & First reported from Reynolds (1981) \\
\hline 87 & Oligonema schweinitzii (Berk.) G. W. Martin & First reported from Macabago et al. (2012) \\
\hline 88 & Perichaena chrysosperma (Curr.) Lister & First reported from Reynolds (1981) \\
\hline 89 & Perichaena corticalis (Batsch) Rostaf. & First reported from Reynolds (1981) \\
\hline 90 & Perichaena depressa Lib. & First reported from Reynolds (1981) \\
\hline 91 & Perichaena dictyonema Rammeloo & First reported from dela Cruz et al. (2014) \\
\hline 92 & Perichaena microspora Penz. \& Lister & First reported from Macabago et al. (2012) \\
\hline 93 & Perichaena minor G. Lister & First reported from Reynolds (1981) \\
\hline 94 & Perichaena pedata (Lister \& G. Lister) Lister ex E. Jahn & First reported from dela Cruz et al. (2011) \\
\hline 95 & $\begin{array}{l}\text { Perichaena reticulospora H.W. Keller \& D.R. } \\
\text { Reynolds }\end{array}$ & First reported from Reynolds (1981) \\
\hline 96 & Perichaena vermicularis (Schwein.) Rostaf. & $\begin{array}{l}\text { First reported from Dagamac et al. (2015a) however } \\
\text { dela Cruz et al. (2014) first reported this species as } \\
\text { Physarum vermiculare Schwein. (Schwein.) Rostaf. }\end{array}$ \\
\hline 97 & Physarella oblonga (Berk. \& M.A. Curtis) Morgan & First reported from Reynolds (1981) \\
\hline 98 & Physaru nucleatum Rex & First reported from Reynolds (1981) \\
\hline 99 & Physarum album (Bull.) Chevall. & First reported as Physarum nutans Pers. by Reynolds (1981) \\
\hline 100 & Physarum bivalve Pers. & First reported from Reynolds (1981) \\
\hline 101 & Physarum bogoriense Racib. & First reported from Reynolds (1981) \\
\hline 102 & Physarum cinereum (Batsch) Pers. & First reported from Reynolds (1981) \\
\hline 103 & Physarum compressum Alb. \& Schwein. & First reported from Reynolds (1981) \\
\hline 104 & Physarum crateriforme Petch & First reported from dela Cruz et al. (2014) \\
\hline 105 & Physarum decipiens M.A. Curtis & First reported from Kuhn et al. (2013a) \\
\hline 106 & Physarum didermoides (Pers.) Rostaf. & First reported from Reynolds (1981) \\
\hline 107 & Physarum echinosporum Lister & First reported from Reynolds (1981) \\
\hline 108 & Physarum flavicomum Berk. & First reported from Reynolds (1981) \\
\hline 109 & Physarum globuliferum (Bull.) Pers. & First reported from Reynolds (1981) \\
\hline 110 & Physarum gyrosum Rostaf. & First reported from Reynolds (1981) \\
\hline 111 & Physarum lakhanpalii Nann.-Bremek. \& Y. Yamam. & First reported from dela Cruz et al. (2014) \\
\hline 112 & Physarum leucophaeum Fr. \& Palmquist & First reported from Dagamac et al. (2010) \\
\hline 113 & Physarum melleum (Berk. \& Broome) Massee & First reported from Reynolds (1981) \\
\hline 114 & Physarum nicaraguense T. Macbr. & First reported from Reynolds (1981) \\
\hline 115 & Physarum notabile T. Macbr. & First reported from Reynolds (1981) \\
\hline 116 & Physarum oblatum T. Macbr. & First reported from Reynolds (1981) \\
\hline 117 & Physarum pezizoideum (Jungh.) Pavill. \& Lagarde & First reported from Reynolds (1981) \\
\hline 118 & Physarum polycephalum Schwein. & First reported from Reynolds (1981) \\
\hline 119 & Physarum psittacinum Ditmar & First reported from Reynolds (1981) \\
\hline 120 & Physarum pulcherrimum Berk. \& Ravenel & First reported from Reynolds (1981) \\
\hline 121 & Physarum pusillum (Berk. \& M.A. Curtis) G. Lister & First reported from Dagamac et al. (2015a) \\
\hline 122 & $\begin{array}{l}\text { Physarum retisporum G.W. Martin, K.S. Thind \& } \\
\text { Rehill }\end{array}$ & First reported from Reynolds (1981) \\
\hline 123 & Physarum rigidum (G. Lister) G. Lister & First reported from Reynolds (1981) \\
\hline 124 & Physarum roseum Berk. \& Broome & First reported from Reynolds (1981) \\
\hline 125 & Physarum rubiginosum Fr. \& Palmquist & First reported from Reynolds (1981) \\
\hline 126 & Physarum stellatum (Massee) G.W. Martin & First reported from Reynolds (1981) \\
\hline 127 & Physarum superbum Hagelst. & First reported from dela Cruz et al. (2014) \\
\hline 128 & Physarum tenerum Rex & First reported from Reynolds (1981) \\
\hline 129 & Physarum viride (Bull.) Pers. & First reported from Reynolds (1981) \\
\hline 130 & Reticularia lycoperdon Bull. & First reported from Reynolds (1981) \\
\hline 131 & Stemonaria fuscoides Nann.-Bremek. \& Y. Yamam. & First reported from Dagamac et al. (2015a) \\
\hline 132 & $\begin{array}{l}\text { Stemonaria longa (Peck) Nann.-Bremek., R.Sharma \& } \\
\text { Y. Yamam. }\end{array}$ & $\begin{array}{l}\text { First reported as Comatricha longa Peck by } \\
\text { Reynolds (1981) }\end{array}$ \\
\hline
\end{tabular}


133 Stemonitis axifera (Bull.) T. Macbr.

134 Stemonitis flavogenita E. Jahn

135 Stemonitis fusca Roth

136 Stemonitis herbatica Peck

137 Stemonitis pallida Wingate

138 Stemonitis splendens Rostaf.

139 Stemonitis uvifera T. Macbr.

140 Stemonitopsis subcaespitosa (Peck) Nann.-Bremek.

141 Stemonitopsis typhina (F.H.Wigg.) Nann.-Bremek.

142 Trichia botrytis (J.F. Gmel.) Pers.

143 Trichia contorta (Ditmar) Rostaf.

144 Trichia decipiens (Pers.) T. Macbr.

145 Trichia erecta $\mathrm{Rex}$

146 Trichia favoginea (Batsch) Pers.

147 Trichia verrucosa Berk.

148 Tubifera ferruginosa Batsch) J.F. Gmel.

149

Tubifera microsperma (Berk. \& M.A. Curtis) G.W. Martin

150
First reported from Reynolds (1981). Stemonitis smithii T. Macbr. was reported as a separate species by Reynolds (1981)

First reported from Dagamac et al. (2015a)

First reported from Reynolds (1981). Stemonitis nigrescens Rex was reported as a separate species by Reynolds (1981)

First reported from Reynolds (1981)

First reported from Dagamac et al. (2010)

First reported from Reynolds (1981)

First reported from dela Cruz et al. (2014)

First reported as Comatricha subcaespitosa Peck by Reynolds (1981)

First reported as Comatricha typhoides (Bull.)

Rostaf. by Reynolds (1981)

First reported from Reynolds (1981)

First reorted as Hemitrichia karstenii (Rostaf.) Lister by Reynolds (1981)

First reported from Reynolds (1981)

First reported from Reynolds (1981)

First reported from Reynolds (1981)

First reported from Reynolds (1981)

First reported from Reynolds (1981)

First reported from Reynolds (1981)

First reported as Cienkowskia reticulata (Alb. \& Schwein.) Rostaf. by Reynolds (1981)

\section{Discussion}

The updated number of myxomycetes records reported for the Philippines is 150 . Comparably this number is still much less than the neotropical country, Costa Rica with 208 recorded species (Rojas et al. 2010). It is of course necessary to point out that Costa Rica is more subjected to much more sampling effort both temporally and spatially. Nevertheless, with regards to the other countries in the Southeast Asian region that were surveyed so far for myxomycetes, this number is considerably more numerous than the species listing data reported for Thailand (132 taxa, Ko Ko et al. 2010), Singapore (92, Rosing et al. 2011), Myanmar (67, Ko Ko et al. 2013), Vietnam (57, Tran et al. 2014) and Laos (44, Ko Ko et al. 2012). Since earlier literature about Philippine myxomycetes were mere species lists and no information about diversity and ecological studies had been conducted in most of the myxomycete surveys, classical approaches of rapid myxomycete surveys were employed at the UST RCNAS (University of Santo Tomas - Research Center for the Natural and Applied Sciences).

\section{Surveys conducted in different forest types in the Philippines}

For most of this survey in the Philippines, a major habitat type investigated for myxomycetes were the lowland mountain forests in the country. Mt. Arayat in Pampanga was used as a model area to observe the occurrence of myxomycetes along an elevational gradient, seasonality and anthropogenic disturbance (Dagamac et al. 2012; Dagamac et al. 2014). The northern slope of Mt. Makulot in Batangas yielded 21 species belonging to 11 genera (Cheng et al. 2013). So far, the highest report of myxomycete diversity for lowland forests were reported in the collective records from Bicol Peninsula with 62 species of myxomycetes reported, of which one species is a first report of Stemonaria fuscoides in the Asian Paleotropics (Dagamac et al. 2015a). National ecoparks in the country were another suitable area for myxomycete surveys because of the intensive environmental protection that at least leads to the development of a secondary type of forest habitat. Baseline information on myxomycete assemblages were conducted in the La Mesa Watershed Ecopark (Macabago et al. 2010), Subic Bay Forest Reserve (dela Cruz et al. 2010), and karst landscape of Quezon National Park (Dagamac et al. 2015b). Furthermore, highland areas were 
also studied by Kuhn et al. 2013b wherein a total of 25 species were reported in Benguet and forest parks in Baguio City.

The coastal forests of the Philippines were another type of habitat that were intensely surveyed since the archipelagic landscape of the Philippines makes it ideal for island biogeographic study of myxomycetes. Among the coastal islands that were already surveyed were several small islands in Hundred Islands National Park and Anda island in Pangasinan (dela Cruz et al. 2011; Kuhn et al. 2013a), Lubang Island in Occidental Mindoro (Macabago et al. 2012) and Polilio Island in Quezon (Viray et al. 2014). Besides simple surveys, comparative assessments of myxomycete diversity based on their forest types were conducted in the recent years. A comparative study between agricultural land and protected forest area of Mt. Kanlaon in Negros Oriental showed that a heterogenous plant community harbored higher myxomycete diversity than monotypic agricultural plantations (Alfaro et al. 2015). Dagamac et al. (2015c) observed how myxomycete communities are similar in terms of composition between mountain forests and coastal forest habitats in Puerto Galera, a UNESCO Man and Biosphere Reserve. Rea-Maminta et al. (2015) also compared myxomycete assemblages from forest patches of the Philippines that were characterized of having either ultramafic or volcanic soils. Such finding is deemed interesting because despite the higher heavy metal content, ultramafic forest patches yielded higher species diversity as compared to volcanic soils.

\section{Opportunities on myxomycete research in the Philippines}

Though admittedly, investigations in terms of ecological patterns for myxomycetes in the Philippines can still be considered in its infancy, several results from different myxomycete surveys have provided interesting insights about the knowledge of Paleotropical myxomycetes. For instance, it has been speculated that myxomycetes follow the intermediate disturbance hypothesis since myxomycete diversity in areas like Bicol Peninsula (Dagamac et al. 2015a) and Puerto Galera (Dagamac et al. 2015c) seem to be affected by either man made activities or natural disturbances. Future research should seek to establish a series of plots along a disturbance gradient in the Philippines to test this hypothesis. In terms of seasonality, the study of Dagamac et al. (2012) in Mt. Arayat National Park is the only one in the country that attempted to compare the diversity of myxomycetes in the clear pronounced wet and dry seasons. The study concluded that the drier season had more species of myxomycetes in comparison to the wet season. It was of course noted that the measures of diversity used in the study were geared more towards standardized substrate sampling design by using solely the moist chamber components. This is due to the fact that erratic rainfalls in the country represent an obstacle in collecting actual fruiting bodies in the field since they can easily be washed away due to strong typhoons that can hit the country during any time of the year. Finding the most appropriate time to search for fruiting bodies in the field is one of the major challenges for myxomycete survey in the Philippines. In order to detect the influence of seasonality on myxomycete diversity, a multi-year survey that utilizes monthly field and substrate collection for moist chambers across a series of standardized plots is recommended. Another aspect that was investigated in Mt. Arayat National Park was the influence of elevation on myxomycete diversity (Dagamac et al. 2014). In that study, no clear pattern was observed since the highest elevation, about 800masl, still had almost the same general vegetation as the lowland elevation (200masl). Such gradients are not as high in comparison to the studies of elevation gradients in Cocos Island, Costa Rica (Rojas \& Stephenson 2008). Investigations on highland areas in the Philippines are still unexplored and such surveys in the future could help fill the missing gaps in the understanding of myxomycete ecology for the Paleotropical Asia.

\section{Other studies that utilized myxomycetes in the country}

Most of the myxomycete diversity studies conducted in the UST - RCNAS included both basic and applied research goals. Field guides for myxomycetes are rare since many tourists and naturalists fail to recognize microorganisms, as such, local interactive keys (Dagamac et al. 2011) and photoguides (Macabago \& dela Cruz 2012) were developed so that myxomycete research could 
at least be accessible for enthusiast and students. The interactive key based on the DELTA software represented the first for myxomycetes in Southeast Asia. Industrial application of myxomycetes is still unpopular but can be of great potential to many possible research endeavors for the future. The successful in vitro culture of myxomycetes could lead to potential mass production of their unknown natural products. Thus, Macabago \& dela Cruz (2014) initialized an attempt to store and preserve cultures of amoeboflagellates from myxomycetes. Moreover, their study had showed that Physarum compressum was able to excrete extracellular enzymes such as amylase and protease. A recent study by Rea-Maminta et al. (2015) reported that myxomycetes could have higher levels of chromium and manganese relative to their substrate. This provides preliminary evidence about the potential of myxomycetes for bioabsorption or bioremediation.

\section{Additional insights and concluding remarks}

Many areas in the world still remained un- or under-studied for myxomycetes (e.g. Madagascar, Wrigley de Basanta et al. 2013; Papua New Guinea and Caledonia, Kylin et al. 2013). This particularly holds true for the Philippines which so farhave covered only 26 provinces of the 81 total provinces or a total of about 20 large islands and small islets from the 7,107 islands the countryis known for. Many habitat types have not been fully explored, e.g. grasslands, mangrove or beach forest, volcanic forest, mossy or cloud forest, high elevation forests dominated by dwarf trees, to name a few. These all represent unique macrohabitats for myxomycetes. The country is also home to more than 6,000 species of endemic plants. If a single species of myxomycetes may be found restricted to a particular substrata or a host plant, then in theory, this could mean that the country could be home to far more species than previously estimated. Reynolds (1981) in his annotated list estimated that the 107 species recorded for the Philippines at that time represented $60 \%$ of the possible species of myxomycetes in the country. Again, if this predication will be accepted as accurate, with the new list of 150 species, adding 43 species in the last 7 years, we are now looking at additional 30 species still waiting to be discovered. Nevertheless, whatever the figures or numbers may be, it is certain that many species of myxomycetes await discovery in the Philippines. Could these unaccounted species be found in many of the unexplored habitats and/or unique substrata in the country? Lastly, the geographic isolation of the Philippines resulted in high endemism among its flora and fauna. Could this also be true for its microbial flora as exemplified by myxomycetes despite their high potential for dispersal? And with some islands previously linked to mainland Asia through the land bridges during the recent and past Ice ages, similarities with some plants and animals in the region have already been documented. Can we also expect similarities in the assemblages of myxomycetes between many parts of Asia and the Philippines? Indeed, the islands of the country offers good sites to explore genetic diversity or study gene flow, speciation, population dynamics or even spore dispersal among myxomycetes. The islands of the Philippines can therefore be a living laboratory to test many hypotheses on key concepts in genetics, ecology and even on interactions between myxomycetes and the endemic host plants or associated insects.

\section{Acknowledgements}

NHAD is grateful for the scholarship support by the DAAD. Authors are also indebted to myxomycete experts, particularly to Prof. Martin Schnittler (EMAU Greifswald) and Prof. Steve L. Stephenson (UARK) who have been very supportive in assisting with establishing myxomycete research in the country. We are also grateful to the anonymous reviewers that helped in improving this manuscript.

\section{References}

Alfaro JRD, Alcayde DLIM, Agbulos JB, Dagamac NHA, dela Cruz TEE. 2015 - The occurrence of myxomycetes from a lowland montane forest and agricultural plantations of Negros Occidental, Western Visayas, Philippines. Fine Focus 1, 7-20. 
Catibog-Sinha CS, Heany L. 2006 - Philippine Biodiversity: Principles and Practise. Quezon City: Haribon Foundation for the Conservation of Natural Resources, Inc.

Cheng CBT, Yu KNT, Campos ML, Adora JMV, Pascua GCP, Pangilinan MVB, Buaya AT, dela Cruz TEE. 2013 - Occurrence and diversity of of myxomycetes (plasmodial slime molds) along the northern slope of Mt. Makulot, Cuenca, Batangas, Philippines. Asian Journal of Biodiversity 4, 65-83.

Dagamac NHA, dela Cruz TEE, Pangilinan MVB, Stephenson SL. 2011 - List of species collected and interactive database of myxomycetes (plasmodial slime molds) for Mt. Arayat National Park, Pampanga, Philippines. Mycosphere 2(4), 449-455.

Dagamac NHA, dela Cruz TEE, Rea-Maninta MAD, Aril-dela Cruz JV, Schnittler M. 2015a Rapid assessment of myxomycete diversity in Bicol Peninsula. Nova Hedwigia doi: http://dx.doi.org/10.1127/nova_hedwigia/2015/0252.

Dagamac NHA, Leontyev DV, dela Cruz TEE. 2010 - Corticolous myxomycetes associated with Samonea samans (Jacq.) Merr. Collected from different sites in Luzon Island, Philippines. The Philippine Biota 43, 2-15.

Dagamac NHA, Rea-Maminta MAD, Batungbacal NS, Jung SH, Bulang CRT, Cayago AGR, dela Cruz TEE. 2015c - Diversity of plasmodial slime molds (myxomycetes) on coastal, mountain, and community forests of Puerto Galera, Oriental Mindoro, Philippines. Journal of Asia Pacific Bioidiversity 8, 322-329.

Dagamac NHA, Rea-Maminta MAD, dela Cruz TEE. 2015b - Plasmodial slime molds of a tropical karst forest, Quezon National Park, the Philippines. Pacific Science 69(3), 407-418.

Dagamac NHA, Stephenson SL, dela Cruz TEE. 2012 - Occurrence, distribution and diversity of myxomycetes (plasmodial slime molds) along two transects in Mt. Arayat National Park, Pampanga, Philippines. Mycology 3, 119-126.

Dagamac NHA, Stephenson SL, dela Cruz TEE. 2014 - The occurrence of litter myxomycetes at different elevations in Mt. Arayat, National Park, Pampanga, Philippines. Nova Hedwigia 98, 187-196.

dela Cruz TEE, Kuhn RV, Javier AOM, Parra CM, and Quimio TH. 2009 - Status of the Myxomycete Collection at the UPLB - Museum of Natural History (UPLB - MNH) Mycological Herbarium. Philippine Journal of Systematic Biology 3, 97-111.

dela Cruz TEE, Kuhn RV, Javier AOM, Rodillas CP, Parra CM, Corpuz LHM, McHugh RD. 2011 - Occurrence and distribution of plasmodial myxomycetes in Hundred Islands National Park, Pangasinan, Philippines. Acta Manilana 59, 65-74.

dela Cruz TEE, Pangilinan MVB, Cruz RJ, de Jesus EE, Puylong RG, Dagamac NHA. 2010 - A checklist of plasmodial myxomycetes (slime molds) from Subic Watershed Forest Reserve, Zambales, Philippines. Acta Manilana 58, 41-45.

dela Cruz TEE, Rea MAD, Tran HTM, Ko Ko TW, Stephenson SL. 2014 - A comparative species listing of myxomycetes from tropical (Philippines) and temperate (United States) forests. Mycosphere 5(2), 299-311.

Dembitsky VM, Rezanka T, Spizek, J, Hanus LO. 2005 - Secondary metabolites of Slime Molds (Myxomycetes). Phytochemistry 66(7), 749-769.

Dogma IJ. 1975 - Of Philippine mycology and lower fungi. Philippine Journal of Biology 4, 69105.

Feest A. 1987 - The quantitative ecology of soil mycetozoa. Progress in Protistology 2, 331-361.

Feest A, Madelin MF. 1988 - Seasonal population changes of myxomycetes and associated organism in four woodland soils. FEMS Microbiology Ecology Ecol 53, 133-140

Foissner W. 1999 - Soil protozoa as bioindicators: pros and cons, methods, diversity, representative sample. Agriculture, Ecosystem \& Environment 74, 95-112.

Hall R. 2002 - Cenozoic geological and plate tectonic evolution of SE Asia and the SWPacific: computer-based reconstructions, model and animations. Journal of Asian Earth Science 20:353-431. 
Heaney LR. 1998 - Vanishing Treasures of the Philippine Rainforests. The Field Museum, University of Chicago Press, Chicago. USA.

Hoppe T, Schnittler M. 2015 - Characterization of myxomycetes in two different soils by TRFLPanalysis of partial 18S rRNA gene sequences. Mycosphere 6(2), 216-227.

Ishibashi M, Iwasaki T, Imai S, Sakamoto S, Yamaguchi K, Ito A. 2001 - Laboratory culture of the myxomycetes: formation of fruiting bodies of Didymium bahiense and its plasmodial production of makaluvamine. Journal of Natural Products 64, 108-110.

Keller HW, Snell KL. 2002 - Feeding activities of slugs on myxomycetes and macrofungi. Mycologia 94(5), 757-760.

Ko Ko TW, Rosing WC, Ko Ko ZZW, Stephenson SL. 2013 - Myxomycetes of Myanmar. Sydowia 65(2), 267-276.

Ko Ko TW, Tran HTM, Clayton ME, Stephenson SL. 2012 - First records of myxomycetes in Laos. Nova Hedwigia 96, 73-81.

Ko Ko TW, Tran HTM, Stephenson SL, Mitchell DW, Rojas C, Hyde KD, Lumyong S. 2010 Myxomycetes of Thailand. Sydowia 62(2), 243-260.

Kobayashi S, Mukai M, Murofushi H, Tigyi G, Murakami- Murofushi K, Uchiyama A, Fujiwara Y, Kobayashi T. 2002 - Biological functions of a novel lipid mediator, cyclic phosphatidic acid. Biochimica et Biophysica Acta 1582, 1-7.

Kuhn RV, Javier AOM, Rodillas CP, Parra CM, Corpus LHM, Moron LS, dela Cruz TEE. 2013b Occurrence and distribution of myxomycetes (plasmodial slime molds) in three provinces of Luzon Island, Philippines. Philippine Science Letter 6, 1-7.

Kuhn RV, Javier AOM, Rodillas CP, Parra CM, Corpuz LHM, Moron LS, dela Cruz TEE 2013a Diversity of plasmodial myxomycetes from Anda Island, Pangasinan, Philippines. Biotropia 20(1), 1-9.

Kylin H, Mitchell DW, Seraoui EH, Buyck B 2013 - Myxomycetes from Papua New Guinea and New Caledonia. Fungal Diversity 59, 33-44

Lado C. 2005-2015 - An on line nomenclatural information system of Eumycetozoa. http://www.nomen.eumycetozoa.com (accessed 10 August 2015).

Lado C, Wrigley de Basanta D, Estrada-Torres A, Stephenson SL 2013 - The biodiversity of myxomycetes in central Chile. Fungal Diversity 59, 3-32

Lantican RM. 2001. The Science and Practice of Crop Production. College, Los Banos, Laguna, Phils.: SEAMEO SEARCA and UPLB. 330 p.

Macabago SAB, Dagamac NHA, dela Cruz TEE. 2010 - Diversity and distribution of plasmodial myxomycetes (slime molds) from La Mesa Ecopark, Quezon City, Philippines. Biotropia $17(2), 51-61$.

Macabago SAB, dela Cruz TEE, Stephenson SL. 2012 - First records of myxomycetes from Lubang Island, Occidental Mindoro, Philippines. Sydowia 64(1), 109-118.

Macabago SAB, dela Cruz TEE. 2012 - Development of a Myxomycete Photoguide as a Teaching Tool for Microbial Taxonomy. Journal of Microbiology and Biology Education. doi: http://dx.doi.org/10.1128/jmbe.v13i1.362.

Macabago SAB, dela Cruz TEE. 2014 - Preservation and extracellular enzyme production of myxomycetes from Lubang Island, Occidental Mindoro, Philippines. Philippine Science Letters 7(2), 331- 336.

Martin G, Alexopoulos CJ. 1969 - The Myxomycetes. University of Iowa Press, Iowa.

Moreno G, Mitchell DW, Stephenson SL, dela Cruz TEE. 2009 - A new species of Craterium (Myxomycetes) with reticulate spores. Boletin Sociedad de Micologia Madrid 33, 175-200.

Philippine Institute for Development Studies. 2005 - Basic on Philippine climatology. Economic Issue of the Day 5 (2): 1-2.

Rea-Maminta MAD, Dagamac NHA, Huyop FZ, Wahab RA, dela Cruz TEE. 2015 - Comparative diversity and heavy metal bisorption of myxomycetes from forest patches on ultramafic and volcanic soils. Chemistry and Ecology 31(8):741-758. 
Reynolds DR. 1981 - Southeast Asian myxomycetes II. Philippines. Philippine Journal of Biology 10 (2-3), 127-150.

Rojas C, Schnittler M, Stephenson SL. 2010 - A review of the Costa Rican myxomycetes (Amoebozoa). Brenesia 73/74, 39-57.

Rojas C, Stephenson SL. 2008 - Myxomycete ecology along an elevational gradient on Cocos Island, Costa Rica. Fungal Diversity 29, 117-127.

Rojas C, Stephenson SL. 2013 - Effect of forest disturbance on myxomycete assemblages in the southwestern Peruvian Amazon. Fungal Diversity 59, 45-53.

Rosing WC, Mitchell DW, Moreno G, Stephenson SL. 2011 - Additions to the myxomycetes of Singapore. Pacific Science 65(3), 391-400.

Schnittler M, Novozhilov YK, Romeralo M, Brown M, Spiegel FW. 2012 - Myxomycetes and myxomycete-like organisms. In: Frey W, ed. Englers syllabus of plant families. Vol. 4. Stuttgart, Germany: Borntra"ger. p 40-88.

Stephenson SL, Stempen H. 1994 - Myxomycetes A Handbook of Slime Molds. USA: Timber Press Inc.

Tran DQ, Nguyen HTN, Tran HTM, Stephenson SL 2014 - Myxomycetes from three lowland tropical forests in Vietnam. Mycosphere 5(5), 662-672.

Uyenco FR. 1973 - Myxomycetes of the Philippines. University of the Philippines Natural Science Research Center Technical Report 12, 1-23.

Viray AT, Rotap DDS, Migraso LL, Sibbaluca NCI, Escobar ECV, Buaya AT, dela Cruz TEE. 2014 - Occurrence and diversity of myxomycetes (slime molds) in Polillo Island, Quezon Province, Philippines. Acta Manilana 62, 9-17.

Wrigley de Basanta D, Lado C, Estrada-Torres A, Stephenson SL 2013 - Biodiversity studies of myxomycetes in Madagascar. Fungal Diversity 59, 55-83 